\title{
Heterozygous Deficiency of Endoglin Decreases Insulin and Hepatic Triglyceride Levels during High Fat Diet
}

\author{
Daniel Beiroa', Amparo Romero-Picó ${ }^{1}$, Carmen Langa ${ }^{2}$, Carmelo Bernabeu², Miguel López ${ }^{1}$, \\ José M. López-Novoa ${ }^{3}$, Ruben Nogueiras ${ }^{1 *}$, Carlos Diéguez ${ }^{1 *}$
}

1 Department of Physiology, School of Medicine-CIMUS - Instituto de Investigaciones Sanitarias (IDIS), CIBER Fisiopatologia de la Obesidad y Nutricion (CIBERobn), University of Santiago de Compostela, Santiago de Compostela, A Coruña, Spain, 2 Centro de Investigaciones Biológicas, Consejo Superior de Investigaciones Científicas (CSIC), and Centro de Investigación Biomédica en Red de Enfermedades Raras (CIBERER), Madrid, Spain, 3 Renal and Cardiovascular Physiopathology Unit, Department of Physiology and Pharmacology, University of Salamanca and Instituto de Investigaciones Biomédicas de Salamanca (IBSAL), Campus Miguel de Unamuno, Salamanca, Spain

\begin{abstract}
Endoglin is a transmembrane auxiliary receptor for transforming growth factor-beta (TGF-beta) that is predominantly expressed on proliferating endothelial cells. It plays a wide range of physiological roles but its importance on energy balance or insulin sensitivity has been unexplored. Endoglin deficient mice die during midgestation due to cardiovascular defects. Here we report for first time that heterozygous endoglin deficiency in mice decreases high fat diet-induced hepatic triglyceride content and insulin levels. Importantly, these effects are independent of changes in body weight or adiposity. At molecular level, we failed to detect relevant changes in the insulin signalling pathway at basal levels in liver, muscle or adipose tissues that could explain the insulin-dependent effect. However, we found decreased triglyceride content in the liver of endoglin heterozygous mice fed a high fat diet in comparison to their wild type littermates. Overall, our findings indicate that endoglin is a potentially important physiological mediator of insulin levels and hepatic lipid metabolism.
\end{abstract}

Citation: Beiroa D, Romero-Picó A, Langa C, Bernabeu C, López M, et al. (2013) Heterozygous Deficiency of Endoglin Decreases Insulin and Hepatic Triglyceride Levels during High Fat Diet. PLoS ONE 8(1): e54591. doi:10.1371/journal.pone.0054591

Editor: Raul M. Luque, University of Cordoba, Spain

Received June 7, 2012; Accepted December 14, 2012; Published January 15, 2013

Copyright: (c) 2013 Beiroa et al. This is an open-access article distributed under the terms of the Creative Commons Attribution License, which permits unrestricted use, distribution, and reproduction in any medium, provided the original author and source are credited.

Funding: This work has been supported by grants from Ministerio de Educacion y Ciencia (CD: BFU2011; ML: RyC-2007-00211; RN: RYC-2008-02219 and SAF200907049; GS: RYC-2009-04972, SAF2010-15881 and SAF2010-19347; JML-N: SAF2010-15881; CB: SAF2010-61827), Xunta de Galicia (ML: 10PXIB208164PR and RN: 2010/14), Junta de Castilla y León (Excellence Group GR100) Fondo Investigationes Sanitarias (ML: PS09/01880), Centro de Investigación Biomédica en Red (CIBER) de Fisiopatología de la Obesidad y Nutrición (CIBERobn), CIBER de Enfermedades Raras (CIBERER) and Red de Investigación Cooperativa en Enfermedades Renales (REDinREN). CIBERobn, CIBERER and RETIC REDinREN are initiatives of the Instituto de Salud Carlos III (ISCIII) of Spain supported by FEDER funds. The research leading to these results has also received funding from the European Community's Seventh Framework Programme under grant agreements (CD, ML and RN: FP7/2007-2013: $n^{\circ}$ 245009: NeuroFAST, and RN: ERC-2011-StG-OBESITY53-281408). The funders had no role in study design, data collection and analysis, decision to publish, or preparation of the manuscript.

Competing Interests: The authors have declared that no competing interests exist.

*E-mail: ruben.nogueiras@usc.es (RN); carlos.dieguez@usc.es (CD)

\section{Introduction}

Endoglin (Eng) is a transmembrane homodimeric glycoprotein $(180 \mathrm{kDa})$ identified in human vascular endothelial cells where it is highly expressed [1]. Eng is also expressed in many other cells types including smooth muscle cells, mesangial cells, fibroblasts, hepatocytes, and keratinocytes [2]. Eng functions as a nonsignaling coreceptor of the transforming growth factor beta (TGF$\beta$ ) modulating its responses $[2,3]$.

Eng modulates processes mainly related to vascular physiology and pathophysiology [2]. Eng plays a key role in endotheliummediated vascular reactivity as it regulates the expression of endothelial nitric oxide synthase (eNOS), and consequently the synthesis of nitric oxide (NO) [4-6] and the expression of cyclooxygenase 2 (COX-2) [7]. Eng expression increases during alterations in vascular structure and function as during embryogenesis, inflammation and wound healing [8] and it is necessary for endothelial cell survival during hypoxia [9]. Eng is required for normal angiogenesis during fetal development as Eng null embryos die at 10-11.5 days due to vascular and cardiac abnormalities [9-11]. Eng also modulates various processes involved in the regulation of angiogenesis in the adult including tumor growth [12-16]. Furthermore, Eng appears involved in the vascular repair carried out by blood mononuclear cells [17] and is associated to hypertension during pregnancy $[18,19]$.

Mutations in the endoglin gene leading to endoglin haploinsufficiency are the cause of the Hereditary Hemorrhagic Telangiectasia (HHT) type 1 [20,21]. Interestingly, gene expression fingerprinting of blood outgrowth endothelial cells demonstrated that compared to healthy subjects, HHT1 patients show $20 \%$ of deregulated genes (upregulated or down regulated) that are involved in metabolic homeostasis [22]. Supporting the link between Eng and metabolism, a relationship between plasma levels of Eng and glycemia was recently found in diabetic patients [23]. In addition, endoglin deficiency is related to endothelial dysfunction [2] and there is a clear association between endothelial dysfunction and alterations in glucose metabolism or metabolic syndrome $[24,25]$. In spite of these evidences, the endogenous role of Eng on energy balance or glucose metabolism is largely unknown. The present study is the first one aimed to investigate the metabolic phenotype of mice haploinsufficient for $\operatorname{Eng}\left(\mathrm{Eng}^{+/-}\right)$ in normal conditions or when challenged with high fat diet. 

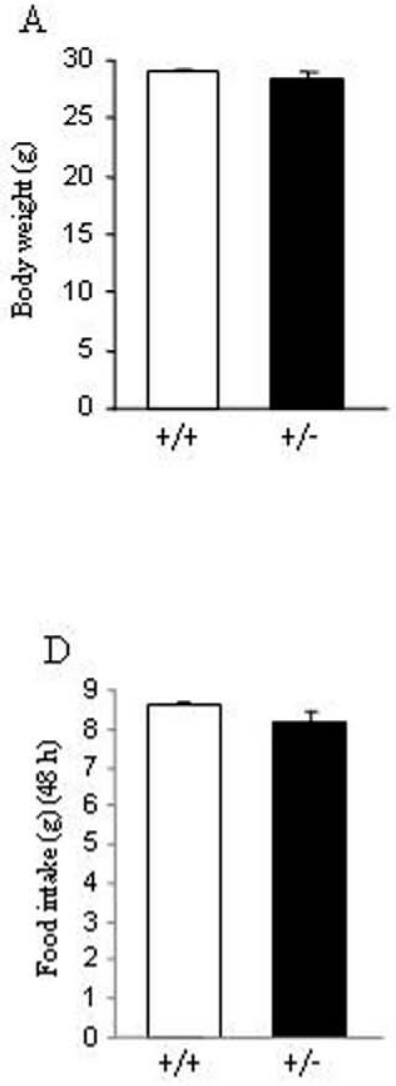

B

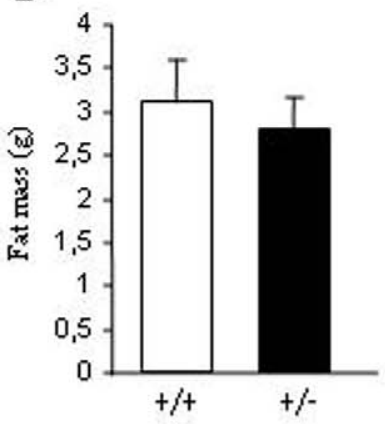

E

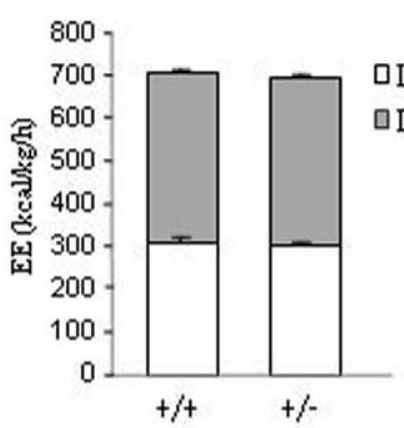

C

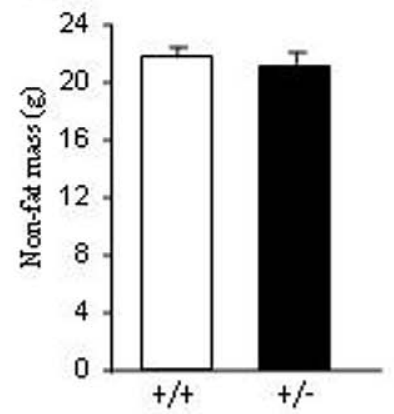

F

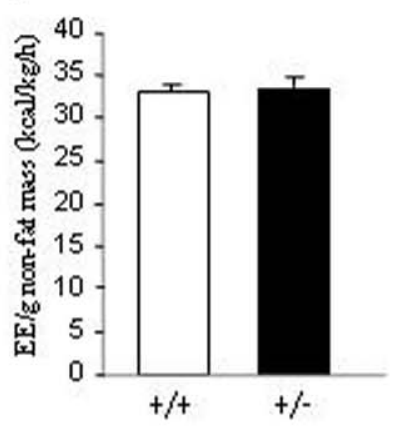

G
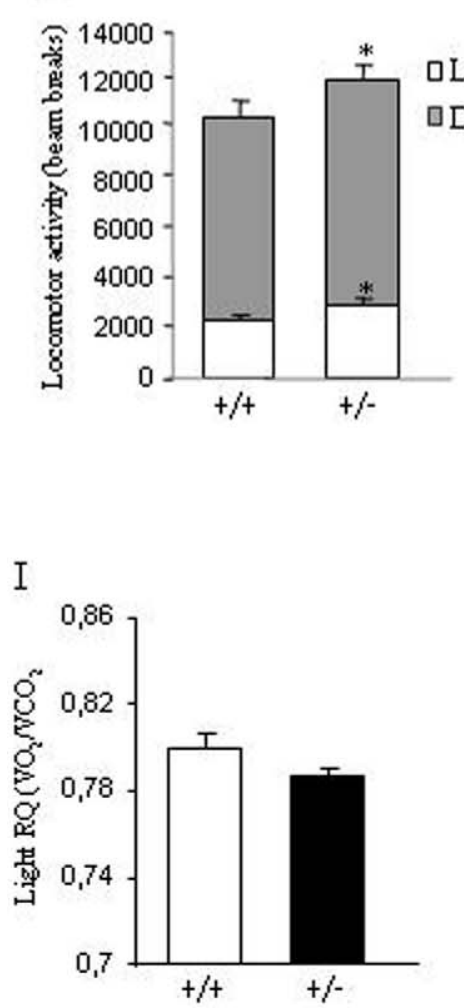

Light Phase

$\mathrm{H}$

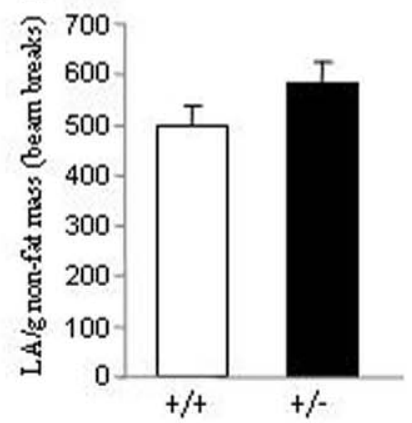

Light Phase

Dark Phase
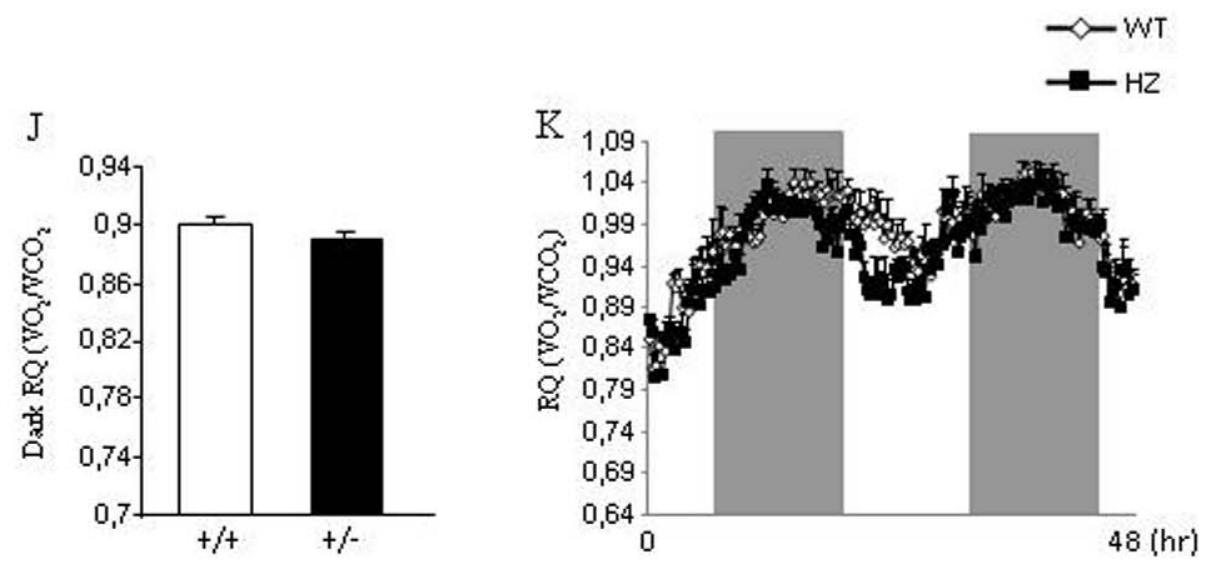
Figure 1. Body weight, body composition, food intake, and metabolic parameters in mice fed a standard diet. Body weight (A), fat mass (B), non-fat mass (C), food intake (D), total energy expenditure (E), energy expenditure corrected by non-fat mass (F), total locomotor activity $(G)$, locomotor activity corrected by non-fat mass $(\mathrm{H})$, respiratory quotient during light phase (I), respiratory quotient during dark phase $(\mathrm{J})$, and $48 \mathrm{~h}$ profile of $\mathrm{RQ}(\mathrm{K})$ in 8-week male wild type and endoglin heterozygous mice fed a standard diet. Measurements were done during $48 \mathrm{~h}$. $\mathrm{n}=6-8$. ${ }^{*} \mathrm{p}<0.05$.

doi:10.1371/journal.pone.0054591.g001

\section{Materials and Methods}

\section{Animals}

Generation and genotyping of $\mathrm{Eng}^{+/-}$mice on a $\mathrm{C} 57 \mathrm{Bl} / 6$ background was previously described $[11,26]$. Mice were kept in ventilated rooms, in a pathogen-free facility under conditions of controlled temperature $\left(23^{\circ} \mathrm{C}\right)$, humidity $(50 \%)$ and illumination (12-hour light/12-hour dark cycle). All studies were performed in parallel in $\mathrm{Eng}^{+/-}$and $\mathrm{Eng}^{+/+}$littermate male mice of 4-6 months of age (20-25 g). After weaning, mice were fed a standard chow diet, and after 8 weeks, the diet was changed to high fat diet (HFD, Research Diets 12451; 45\% fat, 4.73 kcal/g, Research Diets, New Brunswick, NJ) during 16 weeks. All animal procedures performed were approved by the University of Salamanca Animal Care and Use Committee and by the Animal Committee at the University of Santiago de Compostela. All the experiments were performed in agreement with the Rules of Laboratory Animal Care and International Law on Animal Experimentation.

\section{Determination of body composition and energy balance}

Whole body composition was measured using NMR imaging (Whole Body Composition Analyzer; EchoMRI, Houston, TX). Animals were monitored in a custom 12-cage indirect calorimetry, food intake and locomotor activity monitoring system (TSE LabMaster, TSE Systems, Germany) as previously described $[27,28]$. Mice were acclimated for $48 \mathrm{hr}$ to the test chambers and then were monitored for an additional $48 \mathrm{hr}$. Data collected from the last $48 \mathrm{hr}$ was used to calculate all parameters for which results are reported.

\section{Quantitative reverse transcriptase PCR (qRT-PCR) analysis}

RNA was extracted using Trizol ${ }^{\circledR}$ reagent (Invitrogen) according to the manufacturer's instructions and two micrograms of total RNA were used for each RT reaction and cDNA synthesis was performed using SuperScript ${ }^{\text {TM }}$ First-Strand Synthesis System (Invitrogen) and random primers as previously described [29]. Negative control reactions, containing all reagents except the sample were used to ensure specificity of the PCR amplification. For the analysis of gene expression we used real-time reversetranscription polymerase chain reaction (RT-PCR) analyses performed in a fluorescent temperature cycler (TaqMan ${ }^{\circledR}$; Applied Biosystems; Foster City, CA, USA) following the manufacturer's instructions [29,30]. Five hundred ng of total RNA were used for each RT reaction. The PCR cycling conditions included an initial denaturation at $50^{\circ} \mathrm{C}$ for $10 \mathrm{~min}$ followed by 40 cycles at $95^{\circ} \mathrm{C}$ for $15 \mathrm{sec} ; 60^{\circ} \mathrm{C}$ for $1 \mathrm{~min}$. The oligonucleotide specific primers and probes were: G6Pase Fw 5'-CGA GGT CGT GGG TGG AGT CT-3', Rv 5'-TGT AGA TGC CGC GGA TGT G-3', 5' -FAMCAG GCA TTG GTG TGG GTG AAA GTT TCA G-TAM-3'; and PEPCK1 Fw 5'-CCA CAG CTG CTG CAG AAC AC-3', Rv 5'-GAA GGG TCG CAT GGC AAA-3', 5'-FAM-AGG GCA AGA TCA TCA TGC ACG ACG C-TAM- ${ }^{\prime}$. For the analysis of the data, the input value of the gene expression was standardized to the $18 \mathrm{~S}$ value for each sample of each group and was expressed compared with the average value for the control group.

\section{Western blot analysis}

Western blots were performed as previously described [28,31]. Briefly, total protein lysates from liver $(20 \mu \mathrm{g})$, muscle $(20 \mu \mathrm{g})$, and WAT $(15 \mu \mathrm{g})$ were subjected to SDS-PAGE, electrotransferred onto a polyvinylidene difluoride membrane and probed with antibodies against NFkB, PTEN, AKT, pAKT (Ser473), (Cell Signaling, Danvers, MA), and Glut4 (Santa Cruz Biotechnology, Santa Cruz, CA). Recombinant human endoglin tagged with the hemagglutinin (HA) epitope was detected with 12CA5 monoclonal antibody (Roche Diagnostics, Mannheim, Germany). As a loading control, monoclonal antibodies to $\beta$-actin (clone AC-15, Sigma) were used. For primary antibody detection we used horseradish peroxidase-conjugated secondary antibodies and chemiluminescence (Thermo Scientific). We used eight mice per group and the protein levels were normalized to $\beta$-actin for each sample.

\section{Glucose and insulin tolerance tests}

Blood glucose levels were measured with an Accucheck glucometer (Roche) after an intraperitoneal injection of either $2 \mathrm{mg} / \mathrm{g} \mathrm{D}$-glucose (Sigma) or $0.75 \mathrm{U} / \mathrm{kg}$ insulin (Sigma-Aldrich) [32]. Area under the curve (AUC) values were determined and data were analyzed with one-way ANOVA and post-hoc analysis as previously described [27]. GTT and ITT AUC curves were also analyzed with two-way ANOVA using as factors genotype and diet.

\section{TG content in liver}

The extraction procedure for tissue $\mathrm{TG}$ was adapted from methods described previously [28]. Livers (aprox $200 \mathrm{mg}$ ) were homogenized for $2 \mathrm{~min}$ in ice-cold chloroform-methanol (2:1, vol/ vol). TG were extracted during 5-h shaking at room temperature. For phase separation, $\mathrm{H}_{2} \mathrm{SO}_{4}$ was added, samples were centrifuged, and the organic bottom layer was collected. The organic solvent was dried using a Speed Vac and redissolved in chloroform. TG (Randox Laboratories LTD, UK) content of each sample was measured in duplicate after evaporation of the organic solvent using an enzymatic method.

\section{Levels of plasma metabolites and hormones}

Plasma glucose was measured by the glucose oxidase method (Glucose and Triglyceride Spinreact, Spain). Plasma nonesterified fatty acids (NEFA) concentrations were determined using a kit from Wako (US); triacylglycerol (TG) and cholesterol were determined using a kit from Randox Laboratories (LTD, UK). Plasma insulin levels were measured by a previously described RIA [27].

\section{Data Analysis and Statistics}

Values are plotted as the mean \pm SEM for each genotype. Statistical significance was determined by Student's t -test. A P value less than 0.05 was considered statistically significant.

\section{Results}

$\mathrm{Eng}^{+/-}$mice fed a standard diet do not show metabolic alterations

Age-matched male WT and $\mathrm{Eng}^{+/-}$mice of 4 weeks of age were maintained on standard diet for 8 weeks to assess their metabolic 


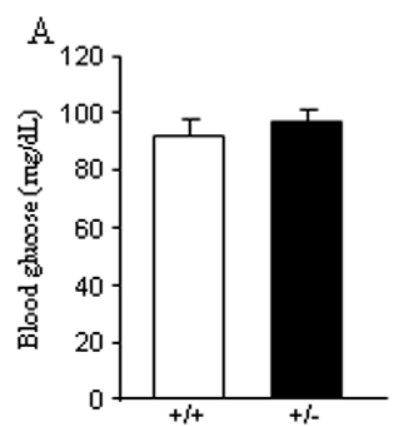

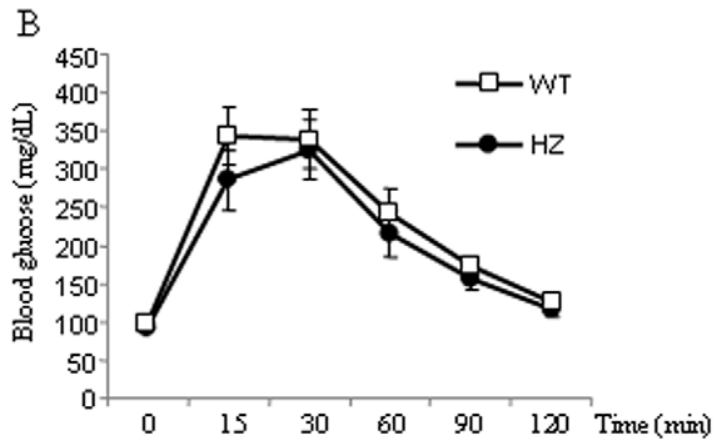

D

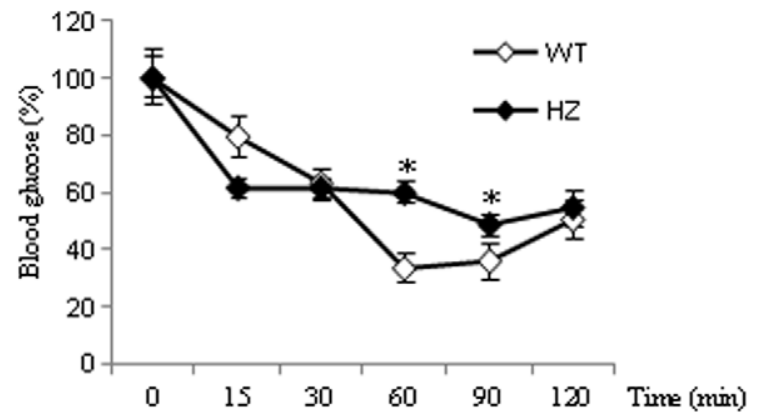

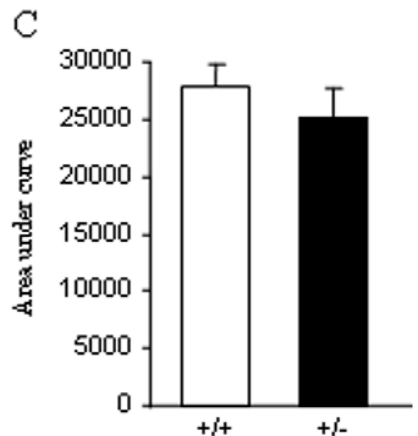

$\mathrm{E}$

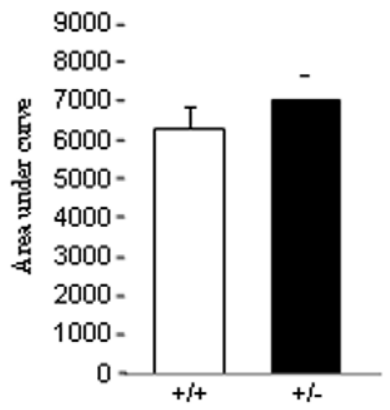

Figure 2. Glucose homeostasis and insulin sensitivity in mice fed a standard diet. Basal glucose levels (A), glucose tolerance test (B), respective area under the curve $(C)$, insulin tolerance test (\% of glucose levels represented against $t 0)(D)$, and respective area under the curve $(E)$ in 8 week male wild type (WT) and endoglin heterozygous (HZ) mice fed a standard diet. $n=6-8$. ${ }^{*} p<0.05$.

doi:10.1371/journal.pone.0054591.g002

phenotypes. No body weight differences were found between both genotypes (Figure 1A). Consistently, body composition (fat mass and non fat mass) (Figure $1 \mathrm{~B}$ and $1 \mathrm{C}$ ) and food intake (Figure 1D) were not altered. Indirect calorimetry was used to determine locomotor activity, energy expenditure and respiratory quotient (RQ). Energy expenditure remained unchanged when WT and $\mathrm{Eng}^{+/-}$mice were fed a standard diet (Figure 1E-1F). Although a slight but significant increase in the locomotor activity of $\mathrm{Eng}^{+/-}$, as compared to WT mice, was observed (Figure 1G), this increase was not found when locomotor activity was corrected by grams of non-fat mass (Figure $1 \mathrm{H})$. In addition, the RQ did not show any statistical difference during the light (Figure $1 \mathrm{I}$ and $1 \mathrm{~K}$ ) or dark phase (Figure 1J and 1K).
Glucose homeostasis in $\mathrm{Eng}^{+/-}$mice fed a standard diet Next, we assessed key parameters of glucose homeostasis in 8 weeks old WT and $\mathrm{Eng}^{+/-}$mice fed a standard diet. This analysis revealed unaltered fasting blood glucose concentrations in $\mathrm{Eng}^{+/-}$mice compared to controls fed a chow diet (Figure 2A). When $\mathrm{Eng}^{+/-}$mice, fed a chow diet, were subjected to intraperitoneal glucose tolerance tests (ipGTT) after an overnight fasting, we did not find any alteration in the tolerance to glucose as compared to WT littermates (Figure 2B-2C). Furthermore, insulin tolerance tests (ITT) after an overnight fasting showed that $\mathrm{Eng}^{+/-}$ mice had increased glucose levels after 60 and $90 \mathrm{~min}$ of insulin injection (Figure 2D). When integrated (area under the curve) 
A

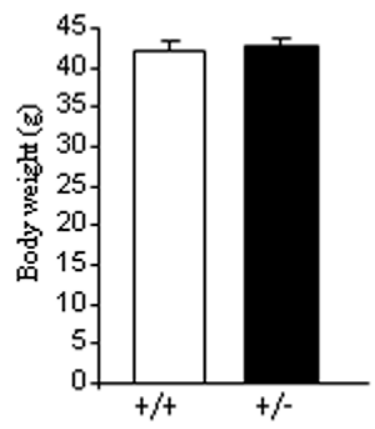

D

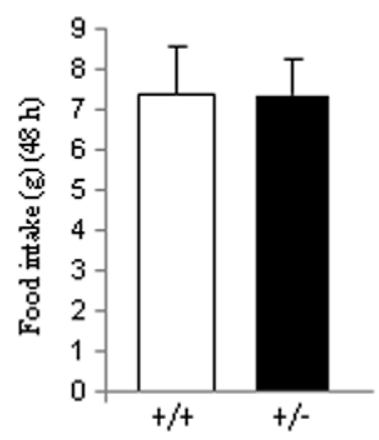

$\mathrm{G}$
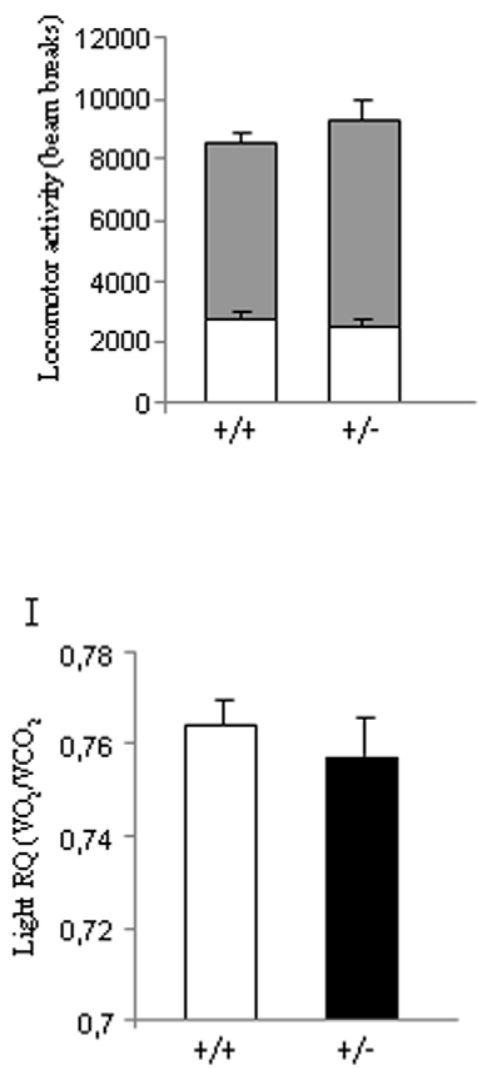

B

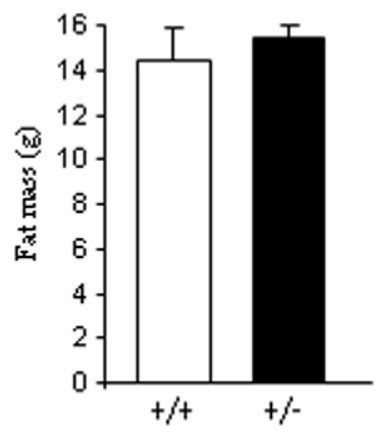

E

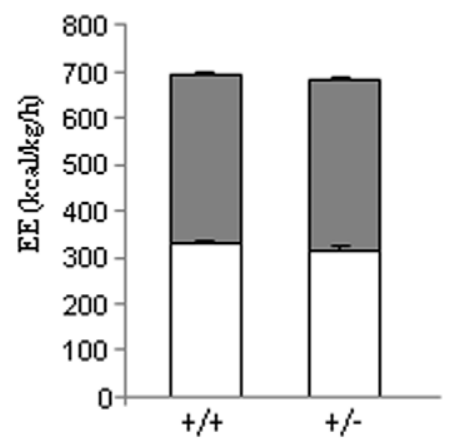

$\mathrm{H}$

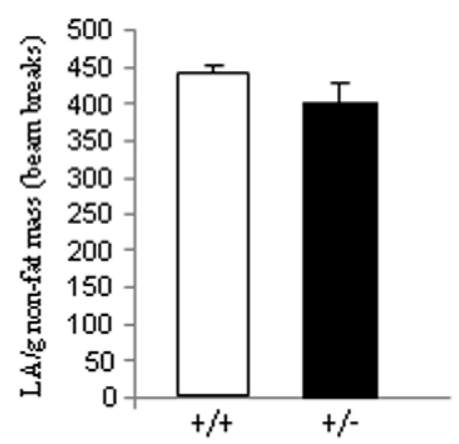

$\mathrm{C}$

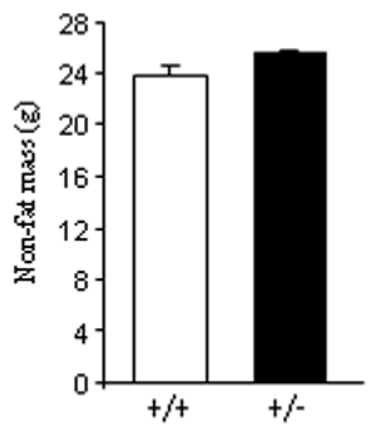

F

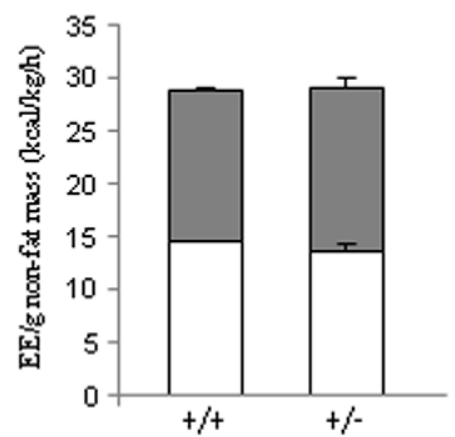

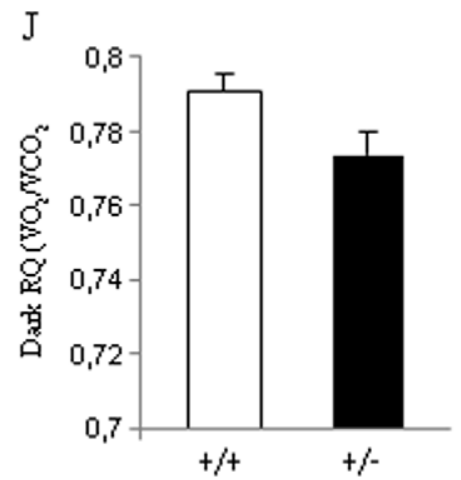

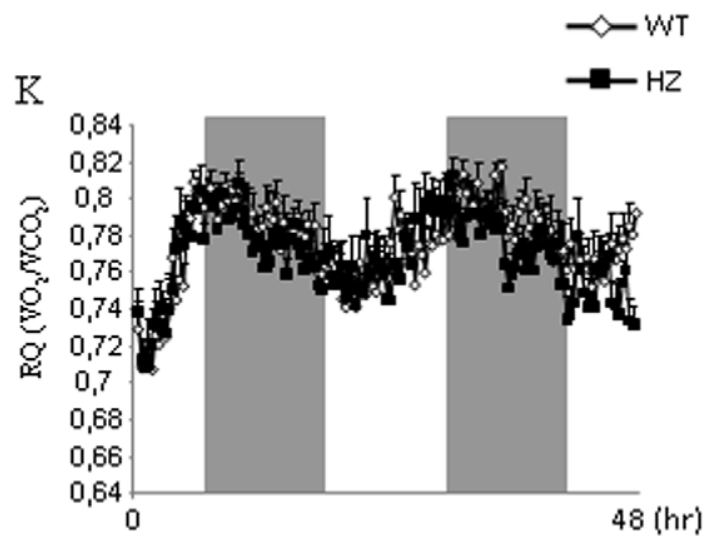


Figure 3. Body weight, body composition, food intake, and metabolic parameters in mice fed a high fat diet. Body weight (A), fat mass $(B)$, non-fat mass (C), food intake (D), total energy expenditure (E), energy expenditure corrected by non-fat mass (F), total locomotor activity $(G)$, locomotor activity corrected by non-fat mass $(\mathrm{H})$, respiratory quotient during light phase $(\mathrm{I})$, respiratory quotient during dark phase $(\mathrm{J})$, and $48 \mathrm{~h}$ profile of RQ $(K)$ in male wild type and endoglin heterozygous mice fed a high fat diet for 16 weeks. Measurements were done during $48 \mathrm{~h}$. $\mathrm{n}=6-8$. doi:10.1371/journal.pone.0054591.g003

glucose levels in $\mathrm{Eng}^{+/-}$mice fed a chow diet failed to show significant differences compared with WT control mice (Figure 2E).

$\mathrm{Eng}^{+/-}$mice fed a HFD show normal body weight and metabolic phenotype

Age-matched male WT and $\mathrm{Eng}^{+/-}$mice of 8 weeks of age were maintained on HFD (45\% kcal fat, $4.73 \mathrm{kcal} / \mathrm{g})$ for $16 \mathrm{wk}$ to assess
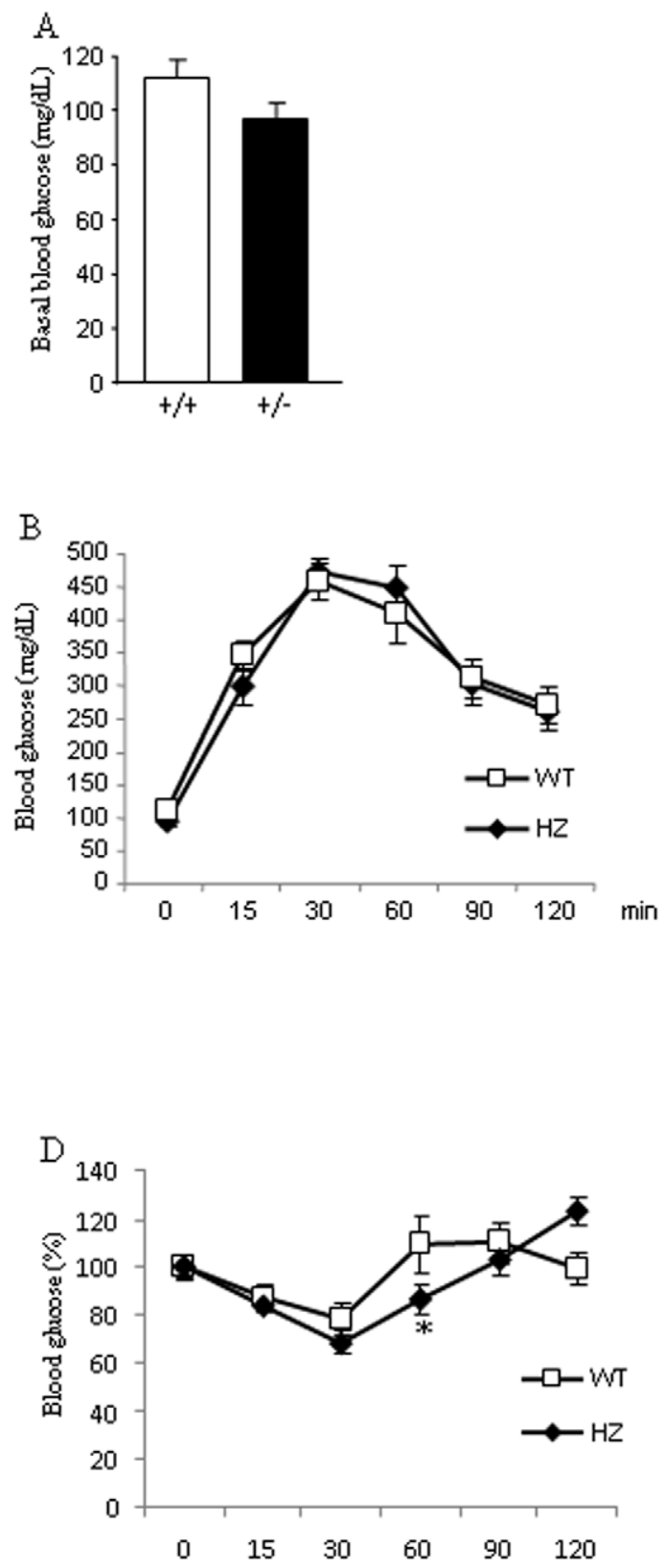

their metabolic phenotypes. No differences in body weight were found between $\mathrm{Eng}^{+/-}$and WT mice when they were fed a HFD (Figure 3A). Consistently, body composition analysis with quantitative NMR revealed that $\mathrm{Eng}^{+{ }^{\prime-}}$ mice fed a HFD gained a similar amount of fat (Figure 3B) and non-fat mass (Figure 3C) compared to WT mice after 16 weeks with HFD. Food intake was also very similar between both genotypes (Figure 3D). Energy expenditure remained unchanged when WT and $\mathrm{Eng}^{+/-}$mice
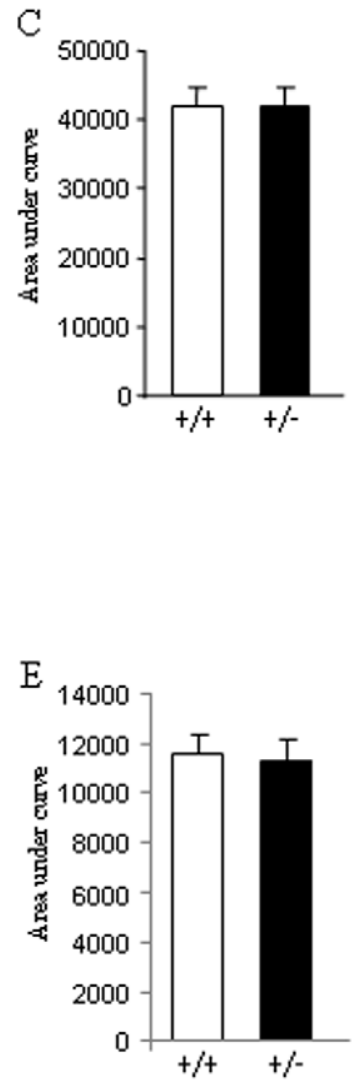

Figure 4. Glucose homeostasis and insulin sensitivity in mice fed a high fat diet. Basal glucose levels (A), glucose tolerance test (B), respective area under the curve $(C)$, insulin tolerance test (\% of glucose levels represented against t0) (D), and respective area under the curve (E) in male wild type (WT) and endoglin heterozygous (HZ) mice fed a high fat diet for 16 weeks. $n=6-8 .{ }^{*} \mathrm{p}<0.05$. doi:10.1371/journal.pone.0054591.g004 

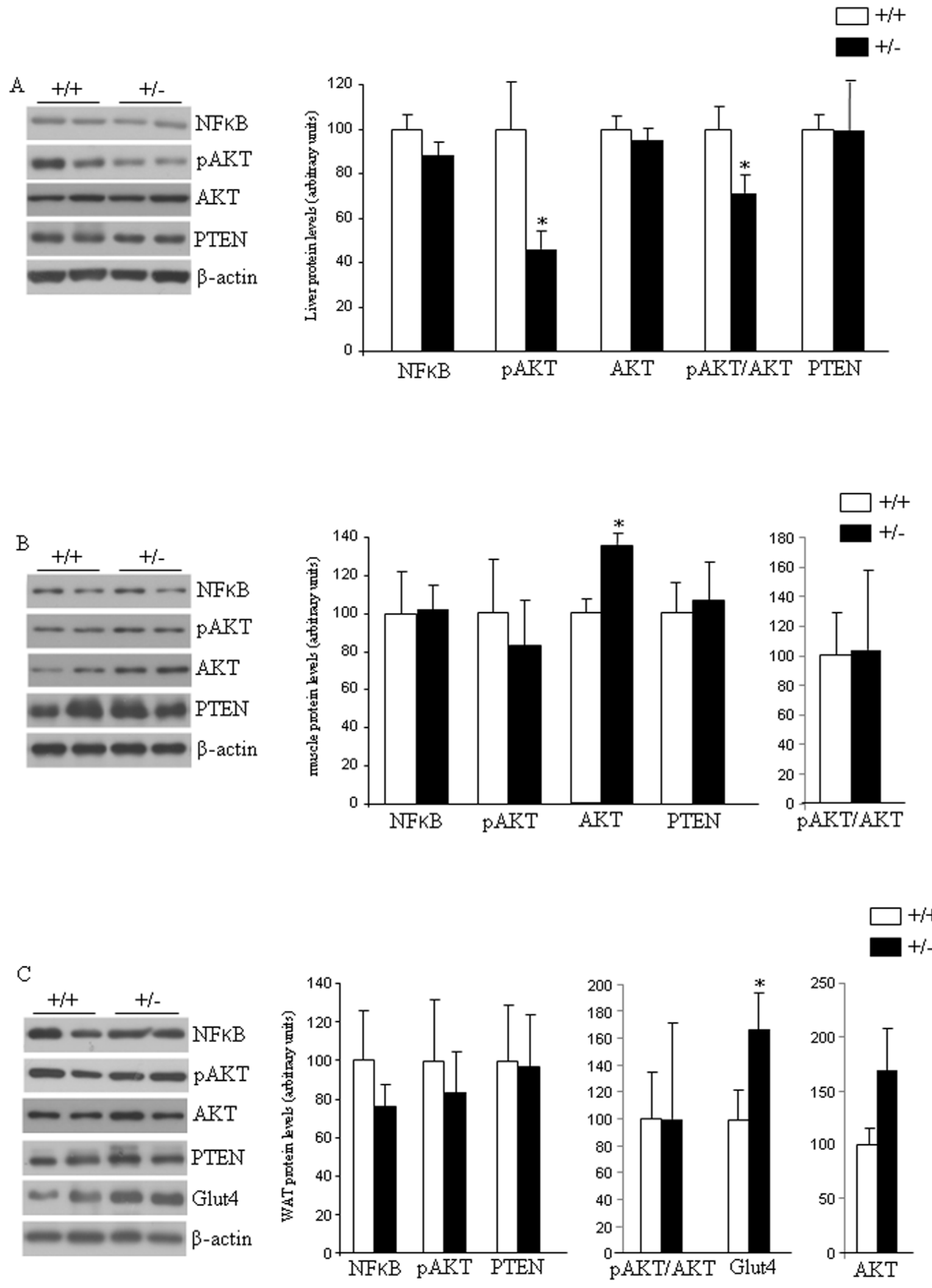
Figure 5. Insulin signaling and glucose uptake in mice fed a high fat diet. Protein levels of NFkB, pAKT, AKT, and PTEN in the liver (A), and muscle (B) of male wild type and endoglin heterozygous mice fed a high fat diet for 16 weeks. Protein levels of NFkB, pAKT (Ser473), AKT, PTEN, and Glut4 in the white adipose tissue (C) of male wild type (+/+) and endoglin heterozygous (+/-) mice fed a high fat diet for 16 weeks. All the samples $(+/+$ and $+/-)$ for each protein were analyzed within the same gel, and the lines represent splicings of the gels. $n=6-8$. ${ }^{*} p<0.05$. doi:10.1371/journal.pone.0054591.g005

were fed a HFD (Figure 3E-3F). No changes were observed in the total locomotor activity between WT and $\mathrm{Eng}^{+/-}$mice (Figure $3 \mathrm{G}$ ). A similar result was observed when locomotor activity was corrected by grams of non-fat mass (Figure 3H). The RQ did
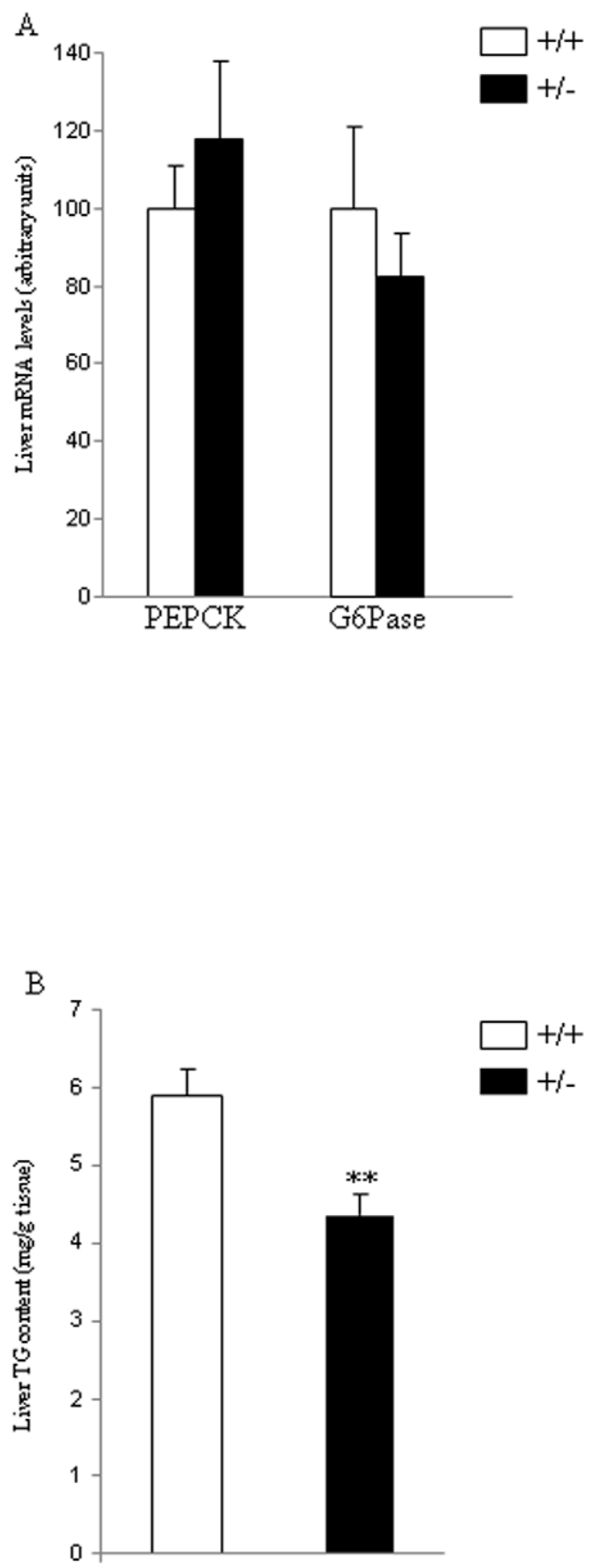

Figure 6. Hepatic glucose production in mice fed a high fat diet. mRNA levels of PEPCK and G6Pase (A) and triglyceride (TG) content (B) in the liver of male wild type and endoglin heterozygous mice fed a high fat diet for 16 weeks. 185 was used as an internal control. $n=6-8 .{ }^{* *} p<0.01$.

doi:10.1371/journal.pone.0054591.g006 not show any statistical difference during the light (Figure 3I and $3 \mathrm{~K}$ ) or dark phase (Figure 3J and 3K).

\section{Glucose homeostasis in $\mathrm{Eng}^{+/-}$mice fed a HFD}

After an overnight fast, plasma glucose levels did not differ between WT and $\mathrm{Eng}^{+/-}$mice fed a HFD (Figure 4A). After an overnight fast, a GTT failed to reveal significant differences in glucose tolerance between both genotypes (Figure 4B-4C). To further investigate the endogenous role of Eng in the control of glucose metabolism, mice were subjected to an ITT after an overnight fast. After $60 \mathrm{~min}$ of insulin injection, glucose values in $\mathrm{Eng}^{+/-}$mice were lower than in WT mice, but no differences were found at the other time points (Figure 4D). When integrated (area under the curve) glucose levels in $\mathrm{Eng}^{+/-}$mice fed a HFD failed to show any significant differences when compared with WT control mice (Figure 4E).

Next, we determined whether Eng interferes with the expression of insulin-signaling components by measuring transcription factor nuclear factor-kappaB (NFkB), pAKT, AKT, and phosphatase and tensin homolog (PTEN) protein levels in liver, skeletal muscle and adipose tissue samples. In the liver we found decreased levels of AKT phosphorylation (Ser473) and the ratio pAKT/AKT, whereas no changes were detected in NFkB, AKT or PTEN (Figure 5A). In muscle, protein levels of these four factors were unaffected by the partial lack of Eng (Figure 5B). In the white adipose tissue (WAT), we also failed to detect changes in protein levels of NFkB, pAKT, AKT, ratio pAKT/AKT, and PTEN (Figure 5G). However, we found increased levels of glucose transporter 4 (Glut4) in the WAT of $\mathrm{Eng}^{+/-}$mice fed a HFD in comparison to WT mice (Figure 5C).

Next, in order to test if the partial lack of Eng was affecting to hepatic glucose production, we measured the mRNA levels of hepatic glucose 6 phosphatase (G6Pase) and phosphoenolpyruvate carboxykinase (PEPGK) (Figure 6A), but failed to detect any change between $\mathrm{Eng}^{+/-}$mice fed a HFD and their littermates. However, triglyceride (TG) content in the liver of $\mathrm{Eng}^{+/-}$mice fed a HFD was significantly lower than in WT mice (Figure 6B).

Then, we measured serum levels of different metabolites and hormones when mice were sacrificed after 16 weeks on HFD. We found that the partial lack of Eng caused a significant decrease in insulin (Figure 7A), but no changes were detected in glucose (Figure 7B), TG (Figure 7C), NEFAs (Figure 7D) or cholesterol (Figure 7E) in WT or $\mathrm{Eng}^{+/-}$mice fed ad libitum.

\section{Discussion}

Mice lacking Eng $\left(\mathrm{Eng}^{-/-}\right)$die during the embryonary phase due to defective angiogenesis $[11,33]$, but $\mathrm{Eng}^{+/-}$mice are viable, breed normally and represent a useful tool to assess the physiological role of endoglin [34]. Here we demonstrate that the partial lack of endoglin in $\mathrm{Eng}^{+/}$mice does not lead to significant changes in body weight, adiposity or food intake. Since HFD induces non-alcoholic fatty liver and causes hyperinsulinemia in mice, a remarkable observation is that after 16 weeks on $\mathrm{HFD}, \mathrm{Eng}^{+/-}$mice showed decreased hepatic triglyceride content and lower insulin levels compared with WT mice.

Eng has emerged as a glycoprotein related with multiple biological actions including angiogenesis, vascular physiology, 
A
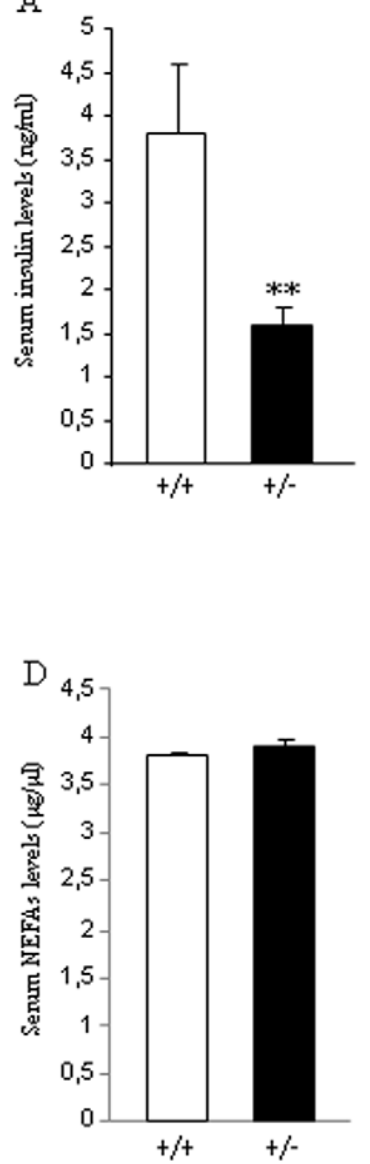

B

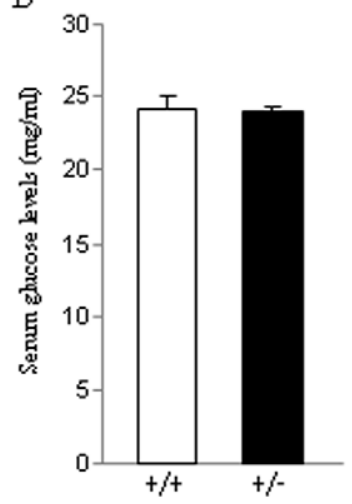

$\mathrm{C}$

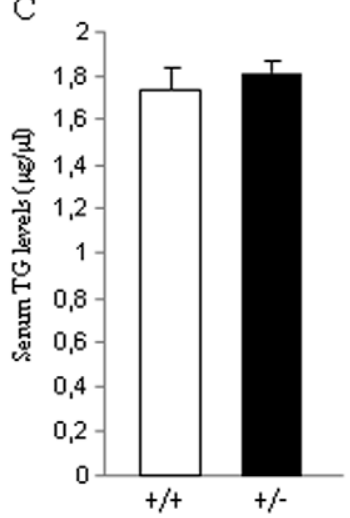

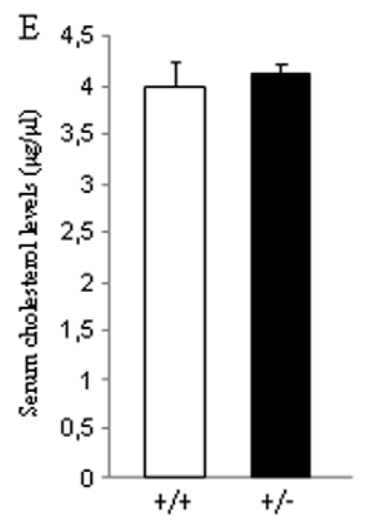

Figure 7. Levels of plasma metabolites and hormones in mice fed a high fat diet. Serum levels of insulin (A), glucose (B), TG (C), NEFAS (D), and cholesterol (E) in male wild type and endoglin heterozygous mice fed a high fat diet for 16 weeks. $n=6-8 .{ }^{* *} p<0.01$. doi:10.1371/journal.pone.0054591.g007

preeclampsia or cancer. Endoglin deficiency causes a decrease in NO synthesis and the subsequent endothelial dysfunction [2], a process usually associated with altered glucose metabolism and metabolic syndrome [24,25]. However, to our knowledge, the potential role of Eng mediating energy homeostasis has been unexplored. Our current findings demonstrate that the heterozygous deficiency of Eng does not cause any important alteration in metabolic parameters affecting body weight regulation. Energy expenditure, locomotor activity, respiratory quotient or feeding behaviour were similar in WT and $\mathrm{Eng}^{+/-}$mice. Importantly, the lack of metabolic phenotype was observed when mice were fed a standard diet or HFD, indicating that endogenous Eng does not seem to be an important player in these metabolic alterations even though mice were challenged to undergo a pathophysiological condition.

Plasma soluble Eng levels have been reported to be positively correlated with basal glycemia in patients with diabetes and hypertension, and with glycated haemoglobin in all patients with diabetes [23]. Therefore, we also investigated the potential relevance of endogenous Eng in glucose metabolism. Our findings indicate that $\mathrm{Eng}^{+/-}$mice fed a standard diet did not show relevant changes in insulin sensitivity in comparison to their control littermates. When mice were challenged with HFD, which increases the risk of obesity and insulin resistance, we observed that insulin levels were significantly decreased when compared to WT mice fed under the same diet. However, insulin sensitivity was similar between $\mathrm{Eng}^{+/-}$and WT mice (Figure 4). Overall, previous data obtained in humans [23] and our current findings in rodents indicate that Eng seem to play an important role in the control of insulin levels, and heterozygous deficiency of Eng decreases HFDinduced hyperinsulinemia. It is important to highlight that this decrease in insulin levels is independent of changes in body weight or adiposity, suggesting that Eng might play a direct role on insulin synthesis and/or secretion. The PI3K/Akt pathway is one of the major downstream targets of the insulin pathway and is negatively regulated by phosphatase and tensin homologue deleted on chromosome 10 (PTEN) [34]. The activation of the PI3K/Akt pathway inhibits the release of soluble Eng from endothelial cells [35]. Conversely, the inhibition of the PI3K/Akt pathway, by overexpression of PTEN stimulates soluble Eng release from endothelial cells [35]. Given the link between Eng and these signaling proteins, we investigated the possibility that PTEN or Akt levels were altered in $\mathrm{Eng}^{+/-}$mice. We detected a significant decrease of pAkt levels in the liver of $\mathrm{Eng}^{+/-}$mice versus WT mice fed a HFD, but not in muscle or WAT. The inverse correlation between endoglin expression and activation of the survival route of Akt in the liver fits well with the anti-apoptotic effect and the active role in endothelial cell proliferation of endoglin [2,9]. Furthermore, PTEN protein levels also remained unmodified between both genotypes. Taken together, these findings suggest that PTEN and Akt are differentially modulated by the partial lack of Eng, and that Akt is regulated in a tissue-specific manner. Cell culture 
and animal studies have also related NF- $\kappa \mathrm{B}$ activity in the pathogenesis of insulin signalling [36]. We failed to detect any significant change in NF- $\mathrm{KB}$ protein levels in the liver, muscle or WAT between WT and $\mathrm{Eng}^{+/-}$mice, indicating that this transcription factor was not regulated by Eng.

At the cellular level, insulin stimulates glucose uptake by inducing the translocation of the glucose transporter 4 (GLUT4) from intracellular storage sites to the plasma membrane, where the transporter facilitates the diffusion of glucose into muscle and adipocytes [37]. Therefore, we assessed the protein levels of the glucose transporters in WAT, and found that Glut4 protein levels were significantly higher in $\mathrm{Eng}^{+/-}$mice fed a HFD when compared to their control littermates. The importance of GLUT4 expression for maintaining glucose homeostasis and insulin sensitivity has been extensively addressed in different animal models [38], and its essential role is reflected by the phenotype caused by the deficiency or over-expression of GLUT4 in mice [39]. Since $\mathrm{Eng}^{+/-}$mice present reduced insulin levels under HFD, the higher levels of Glut4 detected in the WAT of these mice could be a compensatory mechanism to the lower insulin levels. Another key aspect on diet-induced obesity is the increased amount of fatty acids in the liver [40]. Total hepatic TG content was lower in $\mathrm{Eng}^{+/-}$mice than in control mice fed a HFD. Overall, our data might suggest a defect in insulin production in beta cells from $\mathrm{Eng}^{+/-}$mice fed a HFD. Further studies using isolated islets will be necessary to clarify this aspect. Indeed, we cannot rule out the possibility that other factors important for insulin might be also affected in $\mathrm{Eng}^{+/-}$mice. In this regard, a comparative gene expression analysis revealed that in endothelial cells from HHT1 patients, $20 \%$ of the deregulated genes (down or upregulated) respect to cells from healthy subjects were involved in general metabolism [22]. Among these genes it is worth mentioning the presence of several members of the solute carrier (SLG) protein family. This family contains over 300 membrane transport proteins, including the glucose transporters Glut-1 (SLC2A1) and

\section{References}

1. Gougos A, Letarte M (1990) Primary structure of endoglin, an RGD-containing glycoprotein of human endothelial cells. J Biol Chem 265: 8361-8364.

2. Lopez-Novoa JM, Bernabeu C (2010) The physiological role of endoglin in the cardiovascular system. Am J Physiol Heart Circ Physiol 299: H959-974.

3. Cheifetz S, Bellon T, Cales C, Vera S, Bernabeu C, et al. (1992) Endoglin is a component of the transforming growth factor-beta receptor system in human endothelial cells. J Biol Chem 267: 19027-19030.

4. Jerkic M, Rivas-Elena JV, Prieto M, Carron R, Sanz-Rodriguez F, et al. (2004) Endoglin regulates nitric oxide-dependent vasodilatation. FASEB J 18: 609-611.

5. Santibanez JF, Letamendia A, Perez-Barriocanal F, Silvestri C, Saura M, et al. (2007) Endoglin increases eNOS expression by modulating Smad2 protein levels and Smad2-dependent TGF-beta signaling. J Cell Physiol 210: 456-468.

6. Toporsian M, Gros R, Kabir MG, Vera S, Govindaraju K, et al. (2005) A role for endoglin in coupling eNOS activity and regulating vascular tone revealed in hereditary hemorrhagic telangiectasia. Circ Res 96: 684-692.

7. Jerkic M, Rivas-Elena JV, Santibanez JF, Prieto M, Rodriguez-Barbero A, et al. (2006) Endoglin regulates cyclooxygenase-2 expression and activity. Circ Res 99: 248-256.

8. Torsney E, Charlton R, Parums D, Collis M, Arthur HM (2002) Inducible expression of human endoglin during inflammation and wound healing in vivo. Inflamm Res 51: 464-470.

9. Li C, Issa R, Kumar P, Hampson IN, Lopez-Novoa JM, et al. (2003) CD105 prevents apoptosis in hypoxic endothelial cells. J Cell Sci 116: 2677-2685.

10. Arthur HM, Ure J, Smith AJ, Renforth G, Wilson DI, et al. (2000) Endoglin, an ancillary TGFbeta receptor, is required for extraembryonic angiogenesis and plays a key role in heart development. Dev Biol 217: 42-53.

11. Bourdeau A, Dumont DJ, Letarte M (1999) A murine model of hereditary hemorrhagic telangiectasia. J Clin Invest 104: 1343-1351.

12. Jerkic M, Rodriguez-Barbero A, Prieto M, Toporsian M, Pericacho M, et al. (2006) Reduced angiogenic responses in adult Endoglin heterozygous mice. Cardiovasc Res 69: 845-854.

13. Bernabeu C, Lopez-Novoa JM, Quintanilla M (2009) The emerging role of TGF-beta superfamily coreceptors in cancer. Biochim Biophys Acta 1792: 954 973.
Glut-4 (SLC2A4). Thus, in HHT1 cells, electroneutral cation-Cl cotransporter SLC12A2 (Na-K-Cl cotransporter), mitochondrial carrier SLC25A29 (mitochondrial carnitine/acylcarnitine carrier protein CACL), fatty acid transport protein SLC27A3 (fatty acid transport protein 3), nucleoside-sugar transporter SLC35A5 (UDP-sugar transporter protein) and basolateral iron transporter SCL40A1 (ferroportin 1) were downregulated, whereas nucleoside-sugar transporters SLC35B2 (3'-phosphoadenosine 5'-phosphosulfate transporter) and SLC35D3 (fringe connection-like protein 1) were upregulated respect to controls. In addition to the evident involvement of these carrier proteins in the general metabolism, some of them have been reported to be involved in insulin-dependent metabolic pathways [41-43], thus supporting the link between Eng and insulin. Further studies will be necessary to address this issue.

In summary, we conclude that Eng has a physiological role in the regulation of insulin levels and hepatic lipid content, particularly under challenged environmental conditions. The decreased insulin levels and lower hepatic lipid content seem to be independent of changes in body weight or adiposity. These findings expand our knowledge on the physiological effects controlled by Eng, and identify Eng as a potentially important physiological mediator of metabolism.

\section{Acknowledgments}

We thank Dr. Michelle Letarte for giving us the $\mathrm{Eng}^{+/-}$mice and Annette Düwell for the care and genotyping of the laboratory animals.

\section{Author Contributions}

Conceived and designed the experiments: CB ML JML-N RN CD. Performed the experiments: DB AR-P CL. Analyzed the data: DB AR-P CL. Contributed reagents/materials/analysis tools: DB AR-P CL CB ML JML-N RN CD. Wrote the paper: RN CD.

14. Perez-Gomez E, Del Castillo G, Juan Francisco S, Lopez-Novoa JM, Bernabeu C, et al. (2010) The role of the TGF-beta coreceptor endoglin in cancer. ScientificWorldJournal 10: 2367-2384.

15. ten Dijke P, Goumans MJ, Pardali E (2008) Endoglin in angiogenesis and vascular diseases. Angiogenesis 11: 79-89.

16. Nassiri F, Cusimano MD, Scheithauer BW, Rotondo F, Fazio A, et al. (2011) Endoglin (CD105): a review of its role in angiogenesis and tumor diagnosis, progression and therapy. Anticancer Res 31: 2283-2290.

17. van Laake LW, van den Driesche S, Post S, Feijen A, Jansen MA, et al. (2006) Endoglin has a crucial role in blood cell-mediated vascular repair. Circulation 114: 2288-2297.

18. Parrish MR, Murphy SR, Rutland S, Wallace K, Wenzel K, et al. (2010) The effect of immune factors, tumor necrosis factor-alpha, and agonistic autoantibodies to the angiotensin II type I receptor on soluble fms-like tyrosine-1 and soluble endoglin production in response to hypertension during pregnancy. Am J Hypertens 23: 911-916.

19. Gilbert JS, Gilbert SA, Arany M, Granger JP (2009) Hypertension produced by placental ischemia in pregnant rats is associated with increased soluble endoglin expression. Hypertension 53: 399-403.

20. McAllister KA, Grogg KM, Johnson DW, Gallione CJ, Baldwin MA, et al. (1994) Endoglin, a TGF-beta binding protein of endothelial cells, is the gene for hereditary haemorrhagic telangiectasia type 1 . Nat Genet 8: 345-351.

21. Shovlin CL (2010) Hereditary haemorrhagic telangiectasia: pathophysiology, diagnosis and treatment. Blood Rev 24: 203-219.

22. Fernandez LA, Garrido-Martin EM, Sanz-Rodriguez F, Ramirez JR, MoralesAngulo C, et al. (2007) Therapeutic action of tranexamic acid in hereditary haemorrhagic telangiectasia (HHT): regulation of ALK-1/endoglin pathway in endothelial cells. Thromb Haemost 97: 254-262.

23. Blazquez-Medela AM, Garcia-Ortiz L, Gomez-Marcos MA, Recio-Rodriguez JI, Sanchez-Rodriguez A, et al. (2010) Increased plasma soluble endoglin levels as an indicator of cardiovascular alterations in hypertensive and diabetic patients. BMC Med 8: 86.

24. Fornoni A, Raij L (2005) Metabolic syndrome and endothelial dysfunction. Curr Hypertens Rep 7: 88-95. 
25. Hsueh WA, Lyon CJ, Quinones MJ (2004) Insulin resistance and the endothelium. Am J Med 117: 109-117.

26. Rodriguez-Pena A, Eleno N, Duwell A, Arevalo M, Perez-Barriocanal F, et al. (2002) Endoglin upregulation during experimental renal interstitial fibrosis in mice. Hypertension 40: 713-720.

27. Czyzyk TA, Nogueiras R, Lockwood JF, McKinzie JH, Coskun T, et al. (2010) kappa-Opioid receptors control the metabolic response to a high-energy diet in mice. FASEB J 24: 1151-1159.

28. Nogueiras R, Perez-Tilve D, Veyrat-Durebex C, Morgan DA, Varela L, et al. (2009) Direct control of peripheral lipid deposition by CNS GLP-1 receptor signaling is mediated by the sympathetic nervous system and blunted in dietinduced obesity. J Neurosci 29: 5916-5925.

29. Gonzalez CR, Caminos JE, Vazquez MJ, Garces MF, Cepeda LA, et al. (2009) Regulation of visceral adipose tissue-derived serine protease inhibitor by nutritional status, metformin, gender and pituitary factors in rat white adipose tissue. J Physiol 587: 3741-3750.

30. Vazquez MJ, Gonzalez CR, Varela L, Lage R, Tovar S, et al. (2008) Central resistin regulates hypothalamic and peripheral lipid metabolism in a nutritionaldependent fashion. Endocrinology 149: 4534-4543.

31. Velasquez DA, Martinez G, Romero A, Vazquez MJ, Boit KD, et al. (2011) The central Sirtuin 1/p53 pathway is essential for the orexigenic action of ghrelin. Diabetes 60: 1177-1185.

32. Lee SJ, Kim JY, Nogueiras R, Linares JF, Perez-Tilve D, et al. (2010) PKCzetaregulated inflammation in the nonhematopoietic compartment is critical for obesity-induced glucose intolerance. Cell Metab 12: 65-77.

33. Li DY, Sorensen LK, Brooke BS, Urness LD, Davis EC, et al. (1999) Defective angiogenesis in mice lacking endoglin. Science 284: 1534-1537.
34. Mahmoud M, Upton PD, Arthur HM (2011) Angiogenesis regulation by TGFbeta signalling: clues from an inherited vascular disease. Biochem Soc Trans 39: 1659-1666.

35. Cudmore MJ, Ahmad S, Sissaoui S, Ramma W, Ma B, et al. (2012) Loss of Akt activity increases circulating soluble endoglin release in preeclampsia: identification of inter-dependency between Akt-1 and heme oxygenase-1. Eur Heart J 33: $1150-1158$.

36. Arkan MC, Hevener AL, Greten FR, Maeda S, Li ZW, et al. (2005) IKK-beta links inflammation to obesity-induced insulin resistance. Nat Med 11: 191-198.

37. Kahn BB, Flier JS (2000) Obesity and insulin resistance. J Clin Invest 106: 473 481.

38. Pessin JE, Saltiel AR (2000) Signaling pathways in insulin action: molecular targets of insulin resistance. J Clin Invest 106: 165-169.

39. Graham TE, Kahn BB (2007) Tissue-specific alterations of glucose transport and molecular mechanisms of intertissue communication in obesity and type 2 diabetes. Horm Metab Res 39: 717-721

40. Carobbio S, Rodriguez-Cuenca S, Vidal-Puig A (2011) Origins of metabolic complications in obesity: ectopic fat accumulation. The importance of the qualitative aspect of lipotoxicity. Curr Opin Clin Nutr Metab Care 14: 520-526.

41. Wu Q, Ortegon AM, Tsang B, Doege H, Feingold KR, et al. (2006) FATP1 is an insulin-sensitive fatty acid transporter involved in diet-induced obesity. Mol Cell Biol 26: 3455-3467.

42. Casimir M, Lasorsa FM, Rubi B, Caille D, Palmieri F, et al. (2009) Mitochondrial glutamate carrier GC1 as a newly identified player in the control of glucose-stimulated insulin secretion. J Biol Chem 284: 25004-25014

43. Nishi Y, Fujimoto S, Sasaki M, Mukai E, Sato H, et al. (2011) Role of mitochondrial phosphate carrier in metabolism-secretion coupling in rat insulinoma cell line INS-1. Biochem J 435: 421-430. 